\title{
An Analysis of Car and SUV Daytime Parking for Potential Opportunity Charging of Plug-in Electric Powertrains
}

\author{
Danilo Santini, Yan Zhou, Anant Vyas \\ Argonne National Laboratory, 9700 S Cass Ave, Argonne, IL, dsantini@anl.gov
}

The submitted manuscript has been created by UChicago Argonne, LLC, Operator of Argonne National Laboratory (“Argonne”). Argonne, a U.S. Department of Energy Office of Science laboratory, is operated under Contract No. DE-AC02-06CH11357. The U.S. Government retains for itself, and others acting on its behalf, a paid-up nonexclusive, irrevocable worldwide license in said article to reproduce, prepare derivative works, distribute copies to the public, and perform publicly and display publicly, by or on behalf of the Government.

\begin{abstract}
Using the 2009 National Household Transportation Survey (NHTS), an analysis of opportunity charging potential during daytime, for the time interval when a car or SUV is parked for the longest duration, is presented here. We focus on charging at $3.3 \mathrm{~kW}$ or less, either using the charger at the dwelling unit a second time per day (a one charger solution), or using a second charge point at work. Our earlier research with the 2009 NHTS indicates that nearly $60 \%$ of vehicles within this sample were driven to work, or returned to home between 6:00 AM and 6:00 PM. In this analysis we consider the potential for daytime charging before summertime afternoon utility load peaks, anticipating that Public Utility Commissions (PUCs) supporting smart grid pricing strategies may impose much higher electricity costs at these times. We consider $\mathrm{kW}$ ratings of typical opportunity chargers versus overnight chargers. We consider the plug-in hybrid with $28 \mathrm{~km}$ of urban electric range (PHEV28), the extended range electric vehicle with $56 \mathrm{~km}$ of universal all-electric operation capability (EREV56) and the battery electric vehicle with $117 \mathrm{~km}$ of electric range (BEV117). Electricity demand and gasoline fueled miles reduction is examined for the average circumstance in two daily distance brackets $(48-80 \mathrm{~km}, 80-160 \mathrm{~km})$ and for two charging behaviors - (1) overnight and (2) both overnight and during the longest duration parking event of daytime hours, from 6 am to $6 \mathrm{pm}$.
\end{abstract}

Keywords: PHEV, EREV, BEV, electric drive, fast charge

\section{Introduction}

At the present time, plug-in vehicles coming to market are either cars or small SUVs. A charge during the day in addition to the daily overnight charge provides an opportunity to extend a plugin vehicle's daily electrified miles and increase the technology's potential to pay off its added costs by saving more gasoline each day of operation. One deterrent to daytime charging is that advocates of the future smart grid prefer that summer afternoon electricity prices be far higher than overnight prices.

\subsection{Selection of Target Market Subgroups}

In their analysis of interactions of plug-in electric vehicles with the grid, Dallinger and Wietschel $(\mathrm{D} \& \mathrm{~W})$ at the Fraunhofer Institute for Systems and Innovation Research filtered vehicle types in Germany's national vehicle ownership and use survey "Mobility in Germany" for financial suitability [1]. It is stated that PEVs can be characterized by a high annual driving mileage due to economic reasons. ... the investments in PEVs are higher but the operating costs are lower. Technical aspects are also considered. Possible PHEV and $B E V$ users are required to have an assigned parking space. Battery electric vehicles could not substitute for conventional vehicles unless the vehicle was in a multi-vehicle household. Santini et al showed that 
the average vehicle time on the road increased by a factor of three, from about 0.9 to 2.6 hours, when the U.S. 2001 NHTS was divided into sets of vehicles driven less than $80 \mathrm{~km} /$ day and over $80 \mathrm{~km} /$ day [2]. Electric drive was not projected to be financially competitive with conventional powertrains in the shorter daily distance subset. The change in relative financial viability across powertrains investigated was dramatic, indicating that the details should be investigated with more distance subgroups, as is done here.

D\&W conduct a theoretical long-term evaluation [1].They assume infrastructure is available anywhere the vehicle is parked and that the owner will pay attention to pricing signals throughout the day and charge with infinite elasticity, in response to dynamic price signals which change as often as every 15 minutes. Conceptually the analysis assumes voluntary demand response, via indirect controls that may involve automation, but are managed indirectly by consumers themselves rather than directly by utility systems operators. Such a system is currently being developed by General Motors [3] and Google.

\subsection{Charging power}

At the present time in the U.S., the default $\mathrm{kW}$ ratings for the on-board chargers of the Chevrolet Volt Extended Range Electric Vehicle (EREV), Nissan Leaf Battery Electric Vehicle (BEV), and Fisker Karma EREV are 1.4 to $3.3 \mathrm{~kW}$. The coming Ford Fusion Plug-in Electric Vehicle, which will not be capable of all electric drive at U.S. inter-city highway speeds of $120 \mathrm{~km} / \mathrm{h}$, will also reportedly have a standard charger capability of up to $3.3 \mathrm{~kW}$ [4]. This powertrain technology is termed a plug-in hybrid electric vehicle (PHEV). Of the four, only the Nissan Leaf includes a DC fast charge port for up to $50 \mathrm{~kW}$.

"Level 1" charging, via a standard plug in the U.S., on a dedicated circuit, is 1.44 peak $\mathrm{kW}$ when the utility provides $120 \mathrm{~V}$ to a $12 \mathrm{amp}$ circuit (15 amp fuse) and $1.92 \mathrm{~kW}$ with a $16 \mathrm{amp}$ circuit (20 amp fuse). Actual average $\mathrm{kW}$ during charging could be as low as 1.32 and $1.76 \mathrm{~kW}$ respectively, when only $110 \mathrm{~V}$ are available. The latter circumstance is more likely when utilities are experiencing peak load, while the former is more likely during overnight charging. In their study of charging of a compact car and three sizes of SUVs, the Electric Power Research Institute assumed a $1.0 \mathrm{kWh} / \mathrm{hr}$ actual charging rate for the $15 \mathrm{amp}$ circuit and $1.3 \mathrm{kWh} / \mathrm{hr}$ for the
20 amp circuit [5]. Under SAE standards, Level 2 charging can go from 3.3 to $19.2 \mathrm{~kW}$.

In the U.S., even the low end $3.3 \mathrm{~kW}$ choice is an option that usually requires installation of a new circuit and supporting electric vehicle supply equipment (EVSE). "Best case" costs of Level 2 workplace charging retrofits in parking lots have been estimated to be higher than home charging retrofits in garages [6]. Thousands of dollars of costs per charge point are possible in some circumstances, though one would expect that individuals faced with such costs would not purchase a BEV, nor upgrade if they were able to get by with Level 1 charging for an EREV or PHEV. Older U.S. houses without air conditioning could require complete rewiring to support a plug-in electric vehicle with a Level 2 charger. Nissan strongly encourages owners to upgrade to Level 2 EVSE.

Santini observed that there should be a trade-off between the purchase of higher $\mathrm{kW}$ charging capability (allowing repeated charges per day) and battery capacity, to allow very few charges per day to be sufficient [6]. Gnann et al., in this conference, conclude that an increase from $1.44 \mathrm{~kW}$ to $9.6 \mathrm{~kW}$ in the U.S. does not result in a significant increase in the shares of vehicles replaceable by BEVs with the same battery capacities [7]. By looking at some specific details, this paper may help the reader understand why this conclusion was reached. Gnann's results for patterns of driving in Germany (similar in the aggregate to those in the U.S. except for long intercity trips) imply that pack sizes from 5$10 \mathrm{kWh}$ are by far the most desirable for PHEVs. The PHEV and EREV in this paper approximately represent the two ends of the 5-10 kWh bracket, when considering useable $\mathrm{kWh}$. Plotz el al. recommend that further research should include different vehicle sizes and charging infrastructures [8]. This paper certainly supports that conclusion.

A standard charger on-board nearly all currently available plug-in vehicles is capable of either Level 1 or Level 2 charging, up to $3.3 \mathrm{~kW}$. Although they can use the same connector, which meets the Society of Automotive Engineers (SAE) "J1772" standard, cables, circuits and control equipment for Level 2 have to be bigger and more sophisticated than Level 1 due to greater loads and safety requirements.

\subsection{Important International Distinctions}

Default demand response assumptions for plug-in vehicles in Germany and Europe generally are different from the U.S. In contrast to the default Level 1 peak charging rate of 1.44 and $1.92 \mathrm{~kW}$ in 
the U.S., with costly infrastructure upgrades to go to $3.3 \mathrm{~kW}$ and above, for Germany $\mathrm{D} \& \mathrm{~W}$ assumed PHEV chargers with $4 \mathrm{~kW}$ of charging capability (the standard plug has $3.7 \mathrm{~kW}$ ), and BEVs with $8 \mathrm{~kW}$ of charging capability. One question for thought as the reader examines this paper, is whether charge capabilities above 3-4 $\mathrm{kW}$, but less than $19 \mathrm{~kW}$ would be suitable for BEVs to work, or is fast charging at $50 \mathrm{~kW}$ and above necessary? Is $4 \mathrm{~kW}$ and less adequate for PHEVs and EREVs? Are public fast charging investments to allow daytime charging essentially only necessary to address range anxiety questions for BEV owners?

For BEVs, Gnann et al. [7] estimate that $3.7 \mathrm{~kW}$ in Germany and $1.44 \mathrm{~kW}$ in the U.S. are adequate for household charging, with little benefit obtained from higher power. A big factor in variation of infrastructure costs is "behind the wall" when new circuits and or service panels have to be installed in houses. Obviously, in Germany, default plugs in houses will allow faster charging than in the U.S. One might think the lesser daily driving distances in Europe than in the U.S., and the higher default $\mathrm{kW}$ of the local distribution infrastructure there, would make BEVs relatively more feasible in Europe. However, U.S. to German cross-national step from 1.4 to $3.7 \mathrm{~kW}$ in the house does not bring significant benefit for BEV markets. For Germany, Plotz et al. [8] found that BEVs with a given battery capacity could electrify only a slightly larger share of miles than in the U.S. They state that increasing the domestic power levels would not considerably increase the shares of cars that could technically be replaced by a $B E V$.

The lower the $\mathrm{kW}$ rating of the circuit, plug, EVSE and/or charger, the longer it takes to charge a plug-in vehicle. For BEVs, to overcome range anxiety, it is thought that the possibility for rapid high $\mathrm{kW}$ charging is a very important feature in marketability. Unfortunately, this requirement drives up the cost of the BEV and its supporting infrastructure.

\subsection{Effect of Range Limits of BEVs on Probable Markets}

It has recently become common for analysts to lump BEVs, PHEVs and EREVs together with the plug-in electric vehicle (PEV) shorthand. However, it is our contention, and the approach of German analysts that PHEVs and BEVs are so different that they must be examined separately
[8]. D\&W used a consumer survey to arrive at the following $2030 \mathrm{mix}$ of PHEVs and BEVs [1]. According to their scenario, PHEVs dominate over BEVs in terms of numbers of PEVs and share of usable $\mathrm{kWh}$ of battery pack existing in the market.

Table 1 Plug-in electric vehicle types and market shares in D\&W 2030 scenario

\begin{tabular}{|c|c|c|c|c|c|}
\hline $\begin{array}{c}\text { Vehicle } \\
\text { type }\end{array}$ & $\begin{array}{c}\text { Usable } \\
\text { storage } \\
(\mathrm{kWh})\end{array}$ & $\begin{array}{c}\text { Charge } \\
\text { power } \\
(\mathrm{kW})\end{array}$ & $\begin{array}{c}\text { Equivalent } \\
\text { energy use } \\
(\mathrm{kWh} / \mathrm{km})\end{array}$ & $\begin{array}{c}2030 \\
\text { share of } \\
12 \text { million } \\
\text { PEVs }(\%)\end{array}$ & $\begin{array}{c}\text { Share } \\
\text { of PEV } \\
\text { usable } \\
\mathrm{kWh} \\
(\%)\end{array}$ \\
\hline $\begin{array}{c}\text { PHEV } \\
25 \mathrm{~km}\end{array}$ & 4.5 & 4 & 0.18 & 31.6 & 13.2 \\
\hline $\begin{array}{c}\text { PHEV } \\
57 \mathrm{~km}\end{array}$ & 12 & 4 & 0.21 & 50.4 & 56.2 \\
\hline $\begin{array}{c}\text { BEV } \\
100 \mathrm{~km}\end{array}$ & 15 & 8 & 0.15 & 13.9 & 19.4 \\
\hline $\begin{array}{c}\text { BEV } \\
167 \mathrm{~km}\end{array}$ & 30 & 8 & 0.18 & 4.0 & 11.2 \\
\hline
\end{tabular}

\subsection{Battery Cost as a Determinant of Marketability and Plug-in Powertrain Shares}

It has also been observed that as the rate of use of plug-in electric drive vehicles increases, they become more financially competitive with hybrid electric vehicles. When high rates of annual use and frequent charging (once per day, but a high percentage of days per year) and gasoline prices of $\$ 4$ and above combine, then relatively modest plugin hybrids (PHEVs) which operate in blended mode (both the engine and battery provide power as the battery depletes its charge) in high volume production should become financially competitive with HEVs $[2,9]$.

The next level up in the hierarchy of plug-in electric vehicles (not shown in Table 1) is what is called the extended range electric vehicle (EREV) in the U.S., or the range extended electric vehicle in Europe (REEV). These vehicles are capable of operating all electrically when the battery pack is depleting. Generally, they use a "series" powertrain, for which the engine runs a generator that powers a motor to drive the vehicle (there is either no mechanical link between the engine and wheels, or there is a limited use of such a link only at high speeds). The Chevrolet Volt is such a vehicle, as is the Fisker Karma. Finally, there is the pure battery electric vehicle (BEV) that has no engine, but has a very limited range. Propfe et al [10] and Santini et al [2] have estimated that the relative overall costs of operation of PHEVs will be lower than EREVs, which will in turn be lower than BEVs - when they are all charged about once a day. Only amortized 
capital cost and fuel cost were included in [2], while maintenance and depreciation were also included in [10]. Tal and others at UC Davis have examined what might be called a very modest power PHEV in comparison to an EREV and BEV [11]. The "PHEV" configuration that they estimated had very modest battery pack $\mathrm{kWh}$ and $\mathrm{kW}$.

In a 2010 paper titled "Are batteries ready for plug-in hybrid buyers?" Axsen et al. argued that research and development goals of the United States Advanced Battery Consortium (USABC) were too aggressive and a modest PHEV design - which uses more modest power and energy batteries than targeted by the USABC - was probably the right way to start introducing plugin vehicles into the market [12]. They criticized the U.S. battery subsidy for PHEVs, which does not provide any subsidy below 4 nominal $\mathrm{kWh}$ of pack capability, noting that $75 \%$ of consumers who had participated in their "high cost" design game had selected a plug-in hybrid design with less than $2 \mathrm{kWh}$. Shiau et al made a similar argument, estimating that battery pack costs per $\mathrm{kWh}$ would not be low enough to justify anything more than a PHEV $10 \mathrm{~km}$, and even at $\$ 250 / \mathrm{kWh}$, only a PHEV $32 \mathrm{~km}$ could be justified, not a PHEV64 [13].

Aside from Santini et al [2], none of the cited authors folded in an estimate of infrastructure or charge equipment upgrade costs into their evaluation. Many U.S. analysts who have evaluated plug-in vehicles over the years have assumed that PHEVs will be designed to use Level 1 charging without charge equipment upgrades. However, auto designers who intend to market PHEVs worldwide have agreed on a wire-to-vehicle connector design (SAE J1772) that will be compatible with default plugs worldwide. This means that the standard J1772 connector is "overdesigned" relative to the needs of U.S. consumers that have historically been assumed by U.S. academic and research analysts of PHEVs. Axsen et al. did not highlight this. However, in effect, they are advocating the choice of the lowest possible cost option [12]. Shiau et al also did not include any infrastructure costs in their evaluations [13].

The assertions that modest PHEVs should be emphasized are based on the number of consumers that are anticipated to make a selection of a vehicle with a given design, not the number of $\mathrm{kWh}$ of packs sold, nor the rate of utilization of the packs. One might argue that the consumer that pays very little for a plug-in feature is far less likely to use that feature than one that has paid a great deal for the feature. It stands to reason that a consumer who has a high personal preference for all electric drive and is willing to pay the added battery costs to obtain it, would choose to charge as often as possible. A BEV purchaser in a multi-vehicle household would likely try to shift use to that vehicle [14].

Shiau et al did focus on the propensity to use the battery pack in their analysis, demonstrating clearly that the most cost effective use of a PHEV was when the pack was consistently recharged exactly when it was fully depleted, or always before it was depleted (ignoring inconvenience costs of repeated charging in the analysis) [13]. Recharging exactly when a PHEV or EREV pack is empty is, of course, a difficult challenge.

\subsection{Plug-in powertrains chosen for investigation; charging frequency; daily distance and hours driven}

For reasons stated earlier, within the NHTS, we choose to focus on cars and SUVs, assuming that minivans and pickup trucks are not an attractive market [15]. Observing a flattening out of the rapidly declining payback period curve for electric drive at about $48 \mathrm{~km}$ per day in the analysis by Moawad et al, and a maximum intra-urban daily driving distance of about $144 \mathrm{~km}$ in their Kansas City sample, we sliced out observations below 48 $\mathrm{km} /$ day (excessively long payback periods) and above $160 \mathrm{~km} /$ day in this analysis. To help understand the effect of refining the high and low use groups against the prior work of Santini et al [2], we kept the above and below $80 \mathrm{~km}$ cutoff point used there. We show results for each of the four daily driving distance groups (Tables 2 and 3), but only discuss how plug-in electrics might work in the middle two.

We have included the hours of vehicle operation per day in Table 2. The high to low ratio of hours of operation for these distance groups is about 5 . The variation of real annual gasoline prices in the U.S. from 1978 to 2011 involves a high to low value ratio of 2.3 [16]. In other words, when considering the financial viability of a powertrain, it is probably more important to examine hours of use than changes in real fuel prices. If one thinks in terms of hours of use, then the logical way to think about fuel savings is savings per hour of operation. Table 2 clearly shows that the average driving speed of vehicles in the four distance groups increases as distance and hours of operation rise. 
Table 2009 NHTS Statistics for Four Candidate Segments of Daily Driving, By Vehicle Type*

\begin{tabular}{|c|c|c|c|c|c|c|c|c|c|c|c|c|}
\hline Daily km & $\begin{array}{c}\text { All } \\
\text { Autos } \\
\left(10^{6}\right)\end{array}$ & $\begin{array}{c}\text { Cars } \\
\left(10^{6}\right)\end{array}$ & $\begin{array}{c}\text { SUVs } \\
\left(10^{6}\right)\end{array}$ & $\begin{array}{c}\text { Auto km } \\
\left(10^{9}\right)\end{array}$ & $\begin{array}{c}\text { Car km } \\
\left(10^{9}\right)\end{array}$ & $\begin{array}{c}\text { Car } \\
\mathrm{km} / \mathrm{day}\end{array}$ & $\begin{array}{c}\text { Car } \\
\mathrm{km} / \mathrm{hr} .\end{array}$ & $\begin{array}{c}\text { Car } \\
\mathrm{hr} .\end{array}$ & $\begin{array}{c}\text { SUV } \\
\mathrm{km} \\
\left(10^{9}\right)\end{array}$ & $\begin{array}{c}\text { SUV } \\
\mathrm{km} / \\
\text { day }\end{array}$ & $\begin{array}{c}\text { SUV } \\
\mathrm{km} / \mathrm{hr} .\end{array}$ & $\begin{array}{c}\text { SUV } \\
\mathrm{hr} .\end{array}$ \\
\hline $0-48$ & 65.8 & 36.7 & 13.9 & 1.445 & 0.794 & 21.6 & 28.6 & 0.8 & 0.319 & 22.9 & 30.6 & 0.7 \\
\hline $48-80$ & 20.3 & 10.6 & 4.7 & 1.265 & 0.658 & 62.2 & 40.4 & 1.5 & 0.295 & 62.1 & 42.1 & 1.5 \\
\hline $80-160$ & 19.0 & 9.61 & 4.6 & 2.109 & 1.070 & 111 & 49.7 & 2.2 & 0.512 & 111 & 50.3 & 2.2 \\
\hline $160-320$ & 8.1 & 4.31 & 1.7 & 1.756 & 0.935 & 217 & 59.3 & 3.7 & 0.377 & 218 & 60.2 & 3.6 \\
\hline
\end{tabular}

* For vehicles departing from and returning to the dwelling unit

Table 3 Share of Vehicles Vs. Share of Kilometers, by Vehicle Type

\begin{tabular}{|c|c|c|c|c|c|c|}
\hline \multirow{2}{*}{ Daily km } & \multicolumn{2}{|c|}{ Type share (vehicle count) } & \multicolumn{3}{c|}{ Type share (kilometers) } \\
\cline { 2 - 7 } & Cars & SUVs & Other & Cars & SUVs & Other \\
\hline $0-48$ & $60.2 \%$ & $55.6 \%$ & $56 \%$ & $23.2 \%$ & $21.3 \%$ & $20 \%$ \\
\hline $48-80$ & $17.3 \%$ & $19.0 \%$ & $18 \%$ & $19.1 \%$ & $19.7 \%$ & $19 \%$ \\
\hline $80-160$ & $15.6 \%$ & $18.5 \%$ & $18 \%$ & $31.1 \%$ & $34.2 \%$ & $32 \%$ \\
\hline $160-320$ & $6.9 \%$ & $6.9 \%$ & $8 \%$ & $26.6 \%$ & $24.9 \%$ & $29 \%$ \\
\hline
\end{tabular}

In terms of candidate vehicles, we cut the set down from the theoretical six plug-in vehicles in [2], to only three in this case. We examine representative plug-in vehicles that will be available in the U.S. in 2013 - a PHEV with a nominal $28 \mathrm{~km}$ of urban electric range, an EREV with $56 \mathrm{~km}$ of all electric range, and a BEV with $117 \mathrm{~km}$ of all electric range. We deductively examine the trade-offs between purchasing a PHEV28 that is intended to be charged overnight at home and once more during the day, in comparison to an EREV56 that would be charged only overnight. We also consider the circumstance that Shiau et al considered, charging only overnight and driving a PHEV or EREV a significant fraction of the time in charge sustaining mode [13].

Table 3 illustrates the point that capturing the maximum share of vehicles may not be the proper public policy goal if reducing national fuel consumption is the desire. It is very common for analysts to state that the target market is the first group, since the majority of vehicles are driven $80 \mathrm{~km}$ or less and plug-in vehicles with $80 \mathrm{~km}$ or more of range could cover the needs of this segment. Unfortunately, as Moawad et al [9] and Santini et al [2] have shown, these daily driving distances are not a financially lucrative application of electric drive.

Table 2 (and a statistical fit of data in D\&W for Germany) indicates that the average driving speed steadily increases with distance driven. Santini et al [17] noted that, at high average speeds $(77 \mathrm{~km} / \mathrm{h})$ at relative steady flow (i.e using official U.S. "Highway" fuel economy ratings), diesels available in the U.S. saved more fuel per hour of operation compared to conventional gasoline alternatives than did hybrids. Thus, at 77 $\mathrm{km} / \mathrm{h}$ for a PHEV28 or EREV56 charged once a day and driving most miles in charge sustaining mode, at current U.S. costs of operation, if daily miles are consistently above $160 \mathrm{~km}$, electric drive or hybrid drive would likely be financially unfavorable relative to diesel vehicles.

However, we were surprised by the low $60 \mathrm{~km} / \mathrm{h}$ average speed estimated for the $160-320 \mathrm{~km}$ distance interval. In this paper, we cut the top distance of the sample at $320 \mathrm{~km}$, while the Santini et al [2] had used a distance bracket of $80-480 \mathrm{~km}$. The 2001 average speed for that very wide daily distance bracket was $63 \mathrm{~km} / \mathrm{h}$, much higher than the $\sim 45$ $\mathrm{km} / \mathrm{h}$ average for the $80-320 \mathrm{~km}$ grouping here. The average speed of the 114 vehicles in the Kansas City sample used by Moawad et al was $54 \mathrm{~km} / \mathrm{h}$, varying from $32 \mathrm{~km} / \mathrm{h}$ to $86 \mathrm{~km} / \mathrm{h}$ [9]. The $144 \mathrm{~km}$ top daily distance for that sample was less than the top of our NHTS 80-160 km distance interval. Most likely the slower speeds in the NHTS results, and the wider band of distance, are because the NHTS sample vehicles are often used in very large metro areas where congestion and commute distances are worse than in mid-size cities such as Kansas City.

\subsection{Minimizing the cost of daytime charging}

The idea of this paper is to accomplish a second charge per day per plug-in vehicle while installing 
the minimum reasonable amount of charger $\mathrm{kW}$. The thinking is near term, so our conceptual goal is to accomplish as much as possible with known, currently standardized technology available without upgrades to the vehicle. This means use of EVSE which uses connectors to the vehicle based on the SAE J1772 standard. There is widespread controversy over smart meters being installed by utilities across the U.S. Our goal is for plug-in electric vehicles to be sold nationwide with neither a requirement that the owner of the house must adopt a new electric rate structure, nor that a new meter be installed because of the purchase of the plug-in vehicle. In order to avoid the potential for insistence that plug-in vehicle must at some time in the future be sold with a requirement that smart metering be included in the cost, we wish to consider the needs of grid managers (systems operators) to avoid charging during peak periods of demand and to encourage charging when there is plenty of excess generating capacity. We wish to consider the possibility that a plug-in vehicle owner will learn that it is in their interest to voluntarily avoid charging at certain times and to voluntarily charge at other times.

We agree with the statement of A. Maitra of the Electric Power Research Institute that "charging infrastructure must not become a barrier to adoption" [18]. Maitra presented what we will call the charging pyramid. This has also been presented by Zimmerman of General Motors [19]. Later, Zimmerman restated essentially what the charging pyramid shows "PEV charging infrastructure priority is on home charging, followed by workplace charging, followed by public charging - it is important to work strategies and streamline processes in this order" [20]. Maitra notes that it is important to recognize that a $3.3 \mathrm{~kW}$ rating of Level 2 chargers is comparable to whole house summer peak loads in many locations. Similarly analysts at the Electric Power Research Institute and California Public Utilities Commission [21] are indicating concern over plug-in vehicle demand exceeding house electrical peak summer use of 2.0 to $7.7 \mathrm{~kW}$.

Time-of-use (TOU) residential rates are being implemented in many parts of the country, while experiments are being conducted elsewhere. These rates are intended to discourage use of electricity at peak periods and encourage consumption when there is a trough in demand. The time of day and seasonal motivations for these rates can be understood by examining Fig.
1. This figure shows typical weekly load profiles by season for New York, a northern state with relatively cool climate in comparison to Southern California, Arizona, or Texas. Nevertheless, air conditioning causes loads in the summer season to be much higher than in the other three seasons. Maitra shows per house $\mathrm{kW}$ ratings for Connecticut and Virginia at 4.3 and $4.6 \mathrm{~kW}$ respectively, much less than 7.7 $\mathrm{kW}$ in hot Arizona. Crosby shows 6.0-6.5 kW values for inland Southern California, in comparison to 2.0 in cool San Francisco [21]. In Fig. 1, the Tuesday summer peak is nearly twice as high as the winter peak.

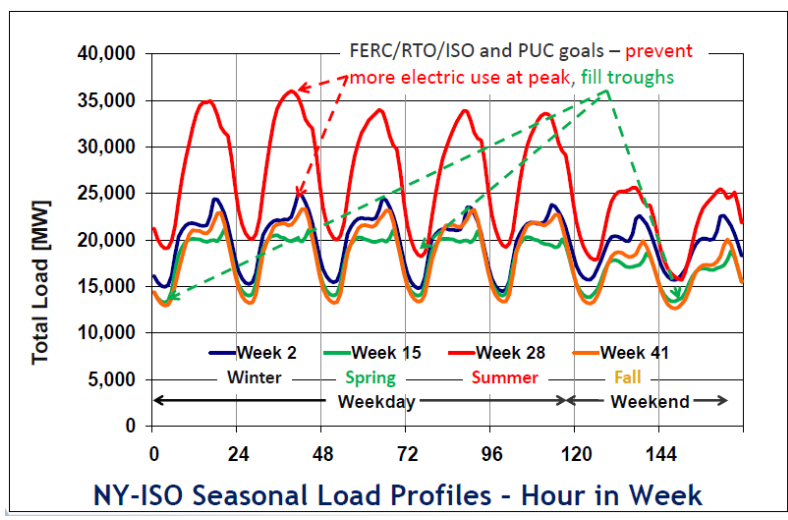

Fig. 1. Typical Weekly Load Profiles by Season, for New York $($ FERC $=$ Federal Energy Regulatory Commission; RTO = Regional Transmission Organization; ISO = Independent System Operator; PUC = Public Utility Commission)

It can be deduced by inspection of Fig. 1 that if a U.S. system of grid, generation, and operators is in place, it will have extra generation capacity on weekends in the summer, and on all days of the remaining four seasons. In all seasons daytime weekday loads are greater than weekend loads, due to business/commercial related loads during weekdays. The effects of the summertime load on the pattern of 2007 time of day rates for Pacific Gas and Electric (PG\&E), the utility serving the San Francisco metro area and surrounding region, are shown in Fig. 2 [22]. One can see that peak rates are charged only during afternoons on weekdays, from 2 $\mathrm{pm}$ to $9 \mathrm{pm}$.

It is also conceivable that installers of Level 2 EVSE might find that if households wished to have the ability to charge their plug-in electric vehicles on hot days with the air conditioner running full blast, it could require a much more expensive upgrade of the wiring and other electrical equipment in the house than if they chose to avoid charging at such times. If customers thinking of pushing the limits of the electrical system capability at their houses were 
made aware that their costs of Level 2 upgrades would be much higher if they want assurance of service on hottest days, while if they did not pay the added installation costs their charging capabilities would be restricted only on hottest days (charging might trip breakers or blow fuses when the air conditioning is most valuable), the problem of peak load charging by plug-in vehicles might end up being of far less concern than smart grid planners think.

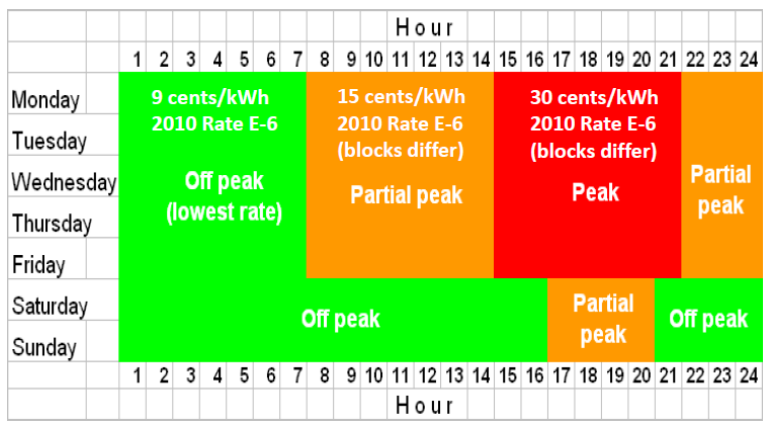

Fig. 2 Pacific Gas and Electric 2007 summer residential rate blocks as a function of time and day

The unknown is what frequency of peak load charging would occur from well informed owners of plug-in vehicles. A concern with batteries is charging when the battery is at high or low temperatures. Neither is desirable. The Nissan Leaf U.S. owner's manual [23] makes it clear that charging when the battery or vehicle are hot is not desirable and may even be disallowed automatically by pack controls. In contrast, the Chevrolet Volt owner's manual does not include such a caution and, in fact says that it is preferable that the vehicle be plugged in when the ambient temperature is above $32^{\circ} \mathrm{C}$ or below $0^{\circ} \mathrm{C}$ [24].

Another general question is whether plug-in electric vehicles are likely to be at the house and be charged on workday summer afternoons. We are able to look at the fraction of vehicles in use that are returned to the house on weekdays and weekends. We have not yet separated out seasonal variation or day of week variation with the 2009 NHTS. However we do note that a very small portion of our sample of vehicles in use includes vehicles that return to the house and would be able to charge at residences on summer afternoons. For our entire sample, we estimate that about $13 \%$ of all vehicles departing from the house in the morning and returning in the evening actually return during the day. Cars and small SUVs are a subset of all vehicles. If these vehicles are owned by multi-vehicle households, some of them might be left at the house and another vehicle preferentially chosen for summer evening use during very hot days, should the owner wish to venture out. The air conditioner would not so easily be turned off nor another appliance substituted for it with little inconvenience. Nevertheless, it might be necessary to impose a very high cost penalty to alter ten days of behavior of a consumer indifferent to peak load problems.

\section{Method}

This paper involves survey of selected literature and deductive interpretation, with limited quantitative illustration using simple algebra. The supporting analysis of the 2009 NHTS follows methods described in $[15,25]$, which are in turn related to methods used in analysis of the 2001 NHTS in Vyas et al [26]. This research refines the prior analysis, reducing the set of vehicles considered by looking only at cars and small SUVs and eliminating daily driving distances less than $48 \mathrm{~km}$ and more than 160 $\mathrm{km}$. We detail trip types and parking durations for this group, isolating the longest duration parking interval as the most logical candidate for one daytime charge. Pursuing financial viability, the sample is restricted to what is thought to be the "heart of the market" - daily use by cars and/or small SUVs in low density dwelling unit types where the vehicles depart from and return to the same parking spot at the dwelling.

\section{Results and Discussion}

\subsection{Work-place and House Charging}

We divided the sample into trip purpose categories. One of the findings is that the duration of parking at work is far longer than at other trip locations (Fig. 3 ). The majority of parking events at work exceed 4 hours, which would allow charging of $13 \mathrm{kWh}$ or more if using a Level 2 charge point at $3.3 \mathrm{~kW}$. This does not fill an empty BEV117, but would easily fill either an empty PHEV28 or EREV56. However, for all other trip types examined in Fig. 3, the vast majority of parking events last less than 2 hours. The $3.3 \mathrm{~kW}$ charge points at such locations would not allow a BEV117 or EREV56 to fully recharge with just one additional daytime charge.

The next best opportunity after the work place is parking at the home, where the share of parking events lasting more than 2 hours exceeds that for any trip type aside from work. Should a plug-in vehicle owner install Level 2 EVSE, then a full recharge of an EREV56 could take place in a little over three hours. The short average durations for home trips 
do imply that a Level 2 charger would be needed to serve our target markets if the owner wanted to avoid repeatedly plugging in during the day, assuming repeated returns to the house allowed for that possibility.

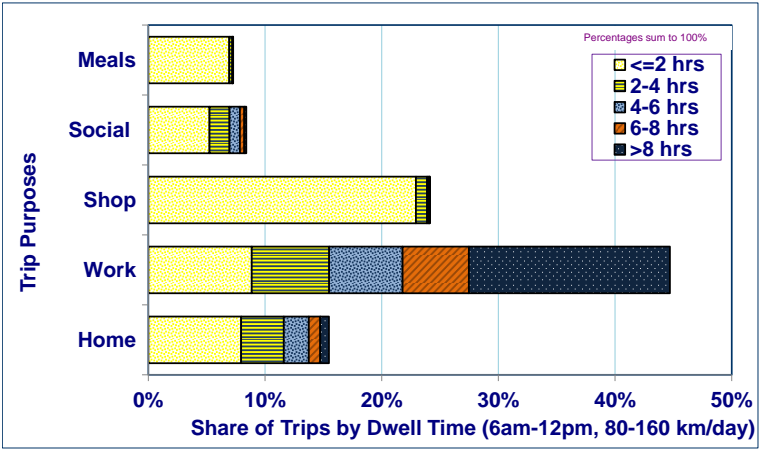

Fig. 3 Duration and Mix of Longest Dwell Times, by Trip Purpose, 6 am to 12 pm (example)

Whether we did the breakout by vehicles or by trips, about $30 \%$ were work use. This is for the entire week. Allowing for the fact that most jobs involve five days of work a week, the share of vehicles used for work would roughly be about $30 \% \times(7 / 5)=42 \%$. However, it is also true that there are not exactly five days of work per week on average, due to vacations and holidays, as well as the fact that some job holders do not work five days a week, and some work from home a portion of the time. Taking these things into consideration we speculated that perhaps half of all vehicles are used during at least part of the week for travel to and from work. On one hand, this implies that workplace charging could enable a second daytime charge for as many as half of the vehicles in the fleet. On the other hand, the fact that workplace parking does not occur at work for five days a week means that a second use at the house would also be important. Unfortunately the NHTS does not allow us to track the use of vehicles for several days, months or more. Thus, the NHTS cannot tell us whether vehicles used for work are more or less likely to be used on non-work days than are other vehicles.

Davies and Kurani [27] recently reported on potential use of PHEVs at work, for a group of 67 sampled households who field evaluated conversion PHEVs over several weeks of use. A subset of $25(37 \%)$ was technically suitable for workplace charging. Weekday and weekend distances of the 25 were recorded for a representative week. By our calculations, assuming five weekdays and two weekend days, weekend distances per day averaged only $35 \%$ of the weekday, which certainly supports the hypothesis that smaller vehicles are used for work and frequently left at the house on weekends as multiple family members share use of a larger vehicle. Also, we cannot know whether vehicles used for work are more or less likely than other vehicles to be away from the house all day on nonwork days when driven far enough to enable use of more than one charge. The Davies and Kurani sample suggests it is logical to assume that weekend days rarely involve exactly the same daily travel distance as on weekdays.

In Germany, where vehicle use sampling follows the same person for an entire week, it has been estimated that optimization based on an assumption that every day of driving is similar to a sample day leads to a significantly greater prediction of successful implementation of electrified miles (by BEVs) than if the entire week is used. At $10 \mathrm{kWh}$, the prediction of BEV suitability dropped from $75 \%$ with an analysis only of daily driving, to $45 \%$ when weekly driving was accounted for. Here the PHEV or EREV has an advantage because it can be driven on the longer trips even if there is no charging infrastructure available where the vehicle will be once the pack is depleted [7].

\subsection{Organization: Arrival Time to Initiate Charging During the Longest Dwell Time of the Day}

With this reservation about the limits of the NHTS sample in mind, we turn to results obtained for vehicles not used for work that do return to the house, and for vehicles used for work. The following charts have a different base for vehicles returning to the house versus those going to work. We include all vehicles returning to the house, out of the entire distance group sample. For the work-trips, we include only cars. We standardize the vertical axis to $8 \%$, the largest share of cars arriving at work in a given hour (i.e. 7-8). By doing so, one sees that even though all vehicles are included in the return to the house case, the share is still considerably less than for the cars driven to work.

Our emphasis is on time of arrival for the longest duration parking event of the day. We break the time intervals into two groups - before and after noon. For each interval we estimate a distance of travel from first departure to the arrival at the location of longest dwell time. This illustrates that if the second charge occurs in the morning, the battery pack may not be depleted before arrival. If it occurs in the evening for a PHEV or EREV, the battery 
may have been depleted, but the remaining travel for the day may not require a full charge.

\section{$48-80 \mathrm{~km}$ daily distance, daytime charging at the dwelling}

In Fig 4, for the 48-80 km distance group it is illustrated that, by count, the majority of longestdwell-time parking events occur in the afternoon, but that the average duration is much less than in the morning. Minor peaking is seen for the 12 pm to 1 pm hour, and 4 pm-5 pm hour. For vehicles that have the longest dwell time in the afternoon, the distance that they have traveled is greater than for the vehicles that have the longest dwell time in the morning. On average these vehicles have gone $44 \mathrm{~km}$, needing to travel 19 $\mathrm{km}$ to complete the day's driving. At 0.22 $\mathrm{kWh} / \mathrm{km}$, they would need about $4 \mathrm{kWh}$. Many would be parked less than 2 hours and would not achieve a $4 \mathrm{kWh}$ charge with about $1.3 \mathrm{~kW}$ in Level 1 charging. Still, if one assumes an average of 2 hours and $2.6 \mathrm{kWh}$ of afternoon charging, a PHEV28 would electrify an equivalent of 40 of $63 \mathrm{~km}$, or $63 \%$. The term "an equivalent of" is used because the PHEVs charge depletion distance can be more variable than for an EREV or EV, depending on the driving behavior of the owner [28]. Aggressive driving of a PHEV can actually lead to more reliance on the engine per $\mathrm{km}$ and extend the distance to depletion, while for an EREV or EV more aggressive driving will always shorten the distance to depletion.

For those vehicles that return in the morning, the problem is that they do not deplete a large fraction of the pack. Nevertheless, the calculations for the PHEV for the day look similar. In this case the vehicles have traveled 24 $\mathrm{km}$ before initiating the daytime charge event. Thus, at our assumed $0.22 \mathrm{kWh} / \mathrm{km}$ they have room for about $5.3 \mathrm{kWh}$. At $1.3 \mathrm{~kW}$, this would require 4.1 hours of charging. Although not all vehicles would have this amount of time available, a significant fraction would. For many of the cases, even with Level 1 charging, the second charge would be over by $2 \mathrm{pm}$, the cost cutoff point in Fig. 2.

Purchasers of PHEVs would have to examine their probable patterns of charging and decide whether the portion of time charging in the late afternoon would make a Level 2 charger desirable. Even if the PHEV owner is faced with a high afternoon summertime rate, the afternoon rate the rest of the year would be much lower. For calculation purposes, assume that the pack is "topped off" and $38 \mathrm{~km}$ of driving would remain. So the PHEV28 would accomplish an equivalent of $85 \%$ of miles electrified. Averaging between the two rough calculations, the PHEV owners could accomplish the equivalent of about 1.6 full charges per day. Even with a charger upgrade, the typical distance at return to the house will either mean the battery is not empty, or the remaining miles of travel in the day will not require a full pack. For this distance group with a PHEV28, upgrades to Level 2 EVSE will seldom make financial sense.

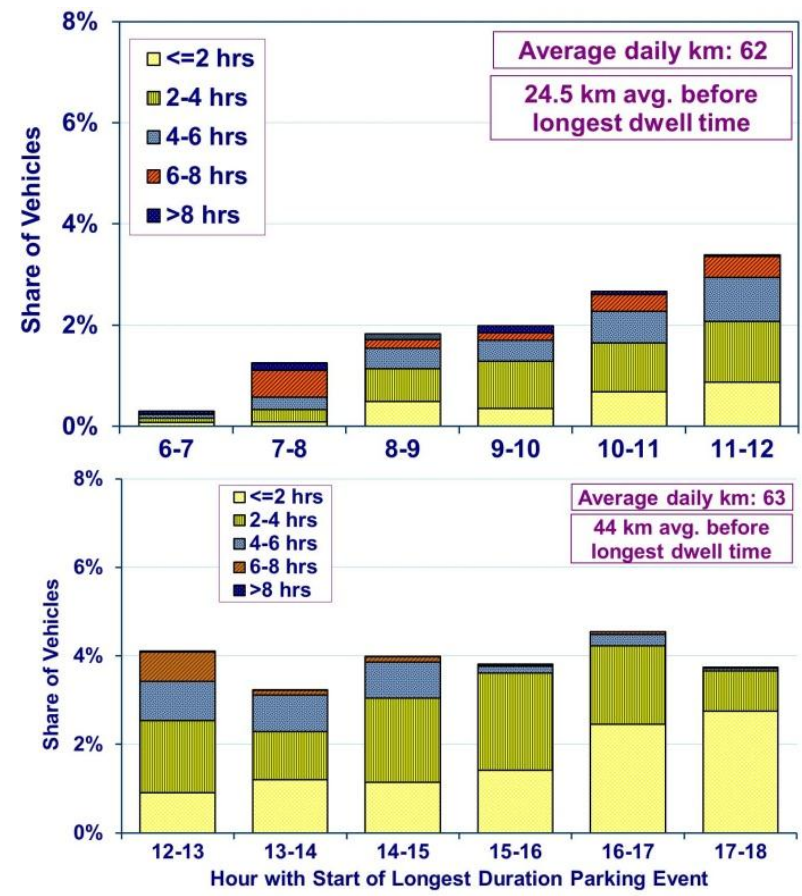

Fig. 4 Share of longest dwell times by hour for morning and afternoon returns to the house (48-80 $\mathrm{km})$

The EREV56 has almost the needed range to serve the average consumer in this group all electrically. On average, a full overnight Level 1 charge would allow all electric driving for almost the entire day. On average, about 1.1 full charge equivalents per day could be accomplished. For this distance group, upgrades to Level 2 charging would also infrequently make financial sense. The BEV would provide more range than needed, so the average number of charges per day of use would only be about 0.5. If applied to this case, the methods of analysis used by Santini et al [2] imply that the inability to accomplish significantly more than one full charge equivalents per day would make the EREV56 and BEV117 financially far less desirable than the PHEV28.

48-80 $\mathrm{km}$ daily distance, daytime charging at work 
The financial viability detriments for the EREV and BEV are primarily a result of the limited daily driving distance in this customer group. Whether the daytime charge opportunity would be at work or at the house would not matter, even if the workplace charger cost nothing. However, from a public policy point of view, it must be recognized that the construction of a second charge point to serve a given vehicle design has to make that vehicle design more costly overall. Unless the charge point (at reasonable cost) allows fills and levels of vehicle utilization that cannot otherwise be accomplished, it should not be built. In this distance group, charging at work could allow more electric driving for EREV56 vehicles in the upper end of this daily distance distribution and nearly twice per day charging for PHEV28s.

The possibility that a workplace charger can cost nothing does exist with Level 1 charge equipment, which is portable and can be carried with the vehicle. Prior research had often tried to estimate the proportion of standard plugs within a reasonable distance of dwelling unit parking places. We assume that the typical work parking location is much further from a plug than typically at parking spots serving dwelling units. Nevertheless, there are work-place parking locations close to plugs. So, some PHEV28 vehicles in this distance group might obtain free charging at work. If they can do so, there is nominal potential to avoid any afternoon charging in the vast majority of cases (Fig. 5).

Nearly $60 \%$ of work trip charging opportunities are for parking events that start before 9 am and last more than four hours. For the $48-80 \mathrm{~km}$ distance group, about $26 \mathrm{~km}$ of distance has been driven on average before work arrival. The battery pack for the PHEV28 that had driven all electrically to work could be nearly filled with Level 1 before $1 \mathrm{pm}$. Thus, for those who arrive at work early and can take advantage of an existing Level 1 plug, their ability to charge would also represent about 1.9 full charges per day of work travel. In this small market the financially superior choice would certainly be a PHEV28, not an EREV56 or BEV117. Although Level 2 charging would not be necessary for these vehicles, the odds are that if a "bank" of new charge points were to be installed in workplace parking lots the points would likely be Level 2, since the incremental cost of Level 2 vs. Level 1 would be small. However, we discuss an alternative possibility below.

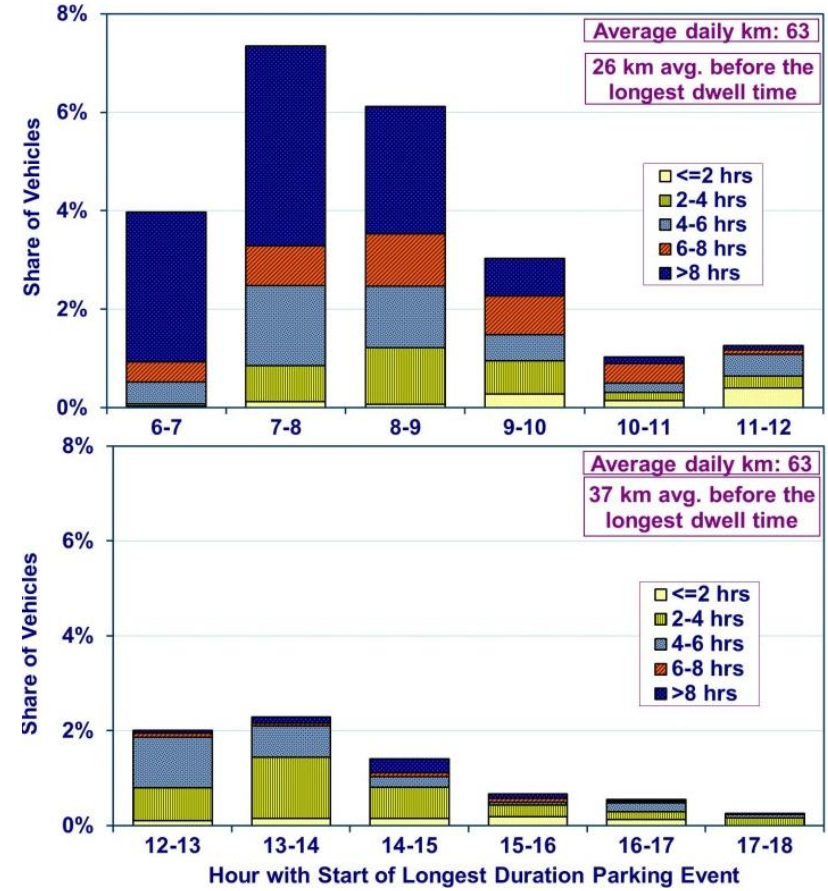

Fig. 5. Share of longest dwell times by hour for cars at work within the $48-80 \mathrm{~km}$ group

Commercial electrical rates are consistently lower than residential rates [21]. Commercial rates generally include a demand charge related to peak $\mathrm{kW}$ use and a fuel charge based on $\mathrm{kWh}$. In many cases, the "fuel charge" rate would be significantly lower than a residential rate. At this time in many states, business and industry may be legally unable to pass electricity costs along to employees using charge points at work. In effect, for commercial customers there has long been an incentive to avoid spikes in their total consumption, which in many cases would also occur on hot summer days. Managers at businesses track their consumption more carefully than the average consumer, partly in order to avoid demand charges. For PHEVs or EREVs, where the vehicle owner has a ready backup in terms of gasoline, businesses might choose to provide electricity free as a "perk" for employees, but simply turn charge points off during days when the business otherwise used a lot of electricity and approached the peak demand allowed under its rate structure. This would probably penalize employees only for a few days during the year.

Conceivably, businesses would choose to adopt Level 1 charging in order to discourage employees from using their personal car for non-work travel. Requiring the plug-in vehicle to remain parked for a long time to fill up, while providing an adequate charge for that employee's return home, could be regarded as strategically smart and "fair". Slower charging would mean that the employee would be 
able to take less advantage of the company's electricity for evening personal use, but still support the employee's work travel. Choosing Level 1 charging would also allow the business to provide the perk to more employees under its demand charge limit, and impose a smaller $\mathrm{kWh}$ penalty per employee if shutting down charge points in the afternoon on hot summer days. Workplace charging may often be offered at a lower cost to the vehicle owner than even for overnight residential charging under Time of Use pricing. In part this would be because the business assumed responsibility for the low voltage distribution of electricity, allowing the utility to avoid this cost.

Davies and Kurani [27] estimated that those plug-in vehicle owners who charged at the workplace might actually cause a higher peak for that fleet in morning hours than overnight hours, noting that those who chose Level 2 charging pushed the peak higher. However, from an overall utility perspective, the question would be what the effect would be from the entire fleet. Elgowainy et al also included an entire fleet charging scenario in which PHEVs at work charged as long as possible and then completed the charging needs for a 24 hour period overnight [25]. In this case also there was a 24 hour peak early in the morning, after arrivals of work vehicles.

If the average arrival distance for afternoon charging is $37 \mathrm{~km}$, then the calculated potential number of charges of a PHEV28 is 1.9, identical to the morning arrival estimate. As was the case for returns to the house, in this distance group, an EREV56 can accomplish about 1.1 charges per day, a BEV117 only 0.5 . This is not an "optimal" distance group to allow the equivalent of multiple charges per day for an EREV56 or BEV117.

This distance bracket is suitable for PHEV28s to charge almost the equivalent of twice per day of use at work. As noted earlier, the weekend and other non-workday opportunities are probably not as attractive (inferences from Davies and Kurani [27]).

\section{0-160 km daily distance}

If a $\mathrm{BEV}$ is to be the solution for this daily distance bracket, the bracket inherently pushes the owner for charge circuit upgrades from the standard U.S. circuit. At $110 \mathrm{~km}$ (Fig. 6) and $0.22 \mathrm{kWh} / \mathrm{km}, 24 \mathrm{kWh}$ are required. At $1.32 \mathrm{~kW}$ for a standard plug, this requires over 18 hours of charging. A Level 1 upgrade to a 20 amp circuit, at $1.76 \mathrm{~kW}$, would require about 14 hours, well above the 10 hour overnight parking period. To allow full charging of a BEV117 once a day, with near depletion each day, would require Level 2 charging (which is what suppliers of BEVs recommend). If the goal of the BEV owner is to take advantage of off-peak charging rates, this would make Level 2 charging even more important, because the duration of off peak periods is usually well under 10 hours.

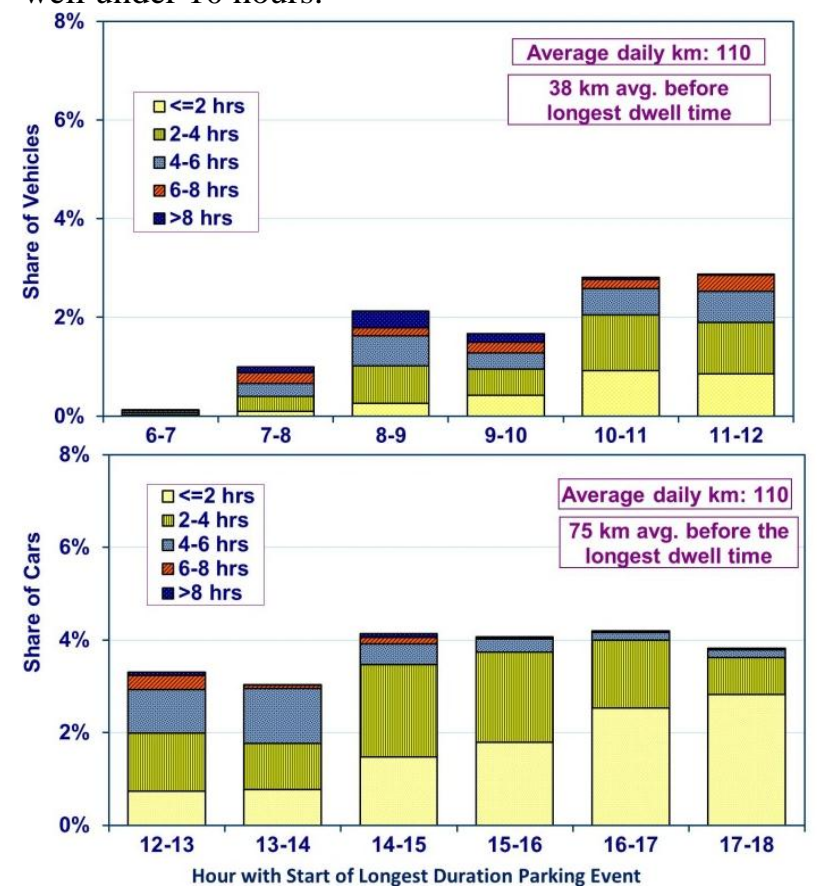

Fig. 6 Share of longest dwell times by hour for morning and afternoon returns to the house $(80-160 \mathrm{~km})$

For an EREV56 and PHEV28, if only one charge per day is to be used, with HEV mode for remaining miles, then Level 1 charging with a 15 amp circuit would allow charging during an off-peak period if such a rate was in force. For an EREV56 an upgrade to a $20 \mathrm{amp}$ circuit would allow charging in about seven hours. By charging a second time each day, the possibility of getting by with Level 1 chargers is enhanced, since the total available charging time per day can be extended relative to the case where the vehicle is only charged overnight. Thus, for this distance bracket, Level 1 charging remains an option when the daytime charge opportunity lasts several hours. As for the prior distance bracket, the two options considered are returning to the parking spot at the dwelling unit, or charging at work.

\section{$80-160 \mathrm{~km}$ daily distance, daytime charging at the dwelling}

Circumstances in the 80-160 km distance group are significantly different. The average daily distance is 
$110 \mathrm{~km}$, far more than twice the charge depleting range of the PHEV28. The average return to the house when the longest dwell time was in the morning would deplete a PHEV28, and the remaining distance to be driven after the average afternoon longest dwell time is also well above $28 \mathrm{~km}$. For the afternoon return, however, the available charge time would often be inadequate to allow a full recharge before departure. For this distance group, for those whose use pattern involves frequent returns to the house, an upgrade to a Level 2 charger becomes more likely, and a desire for afternoon use of the charger also becomes more likely. For this market niche, summertime peak charging rates will affect financial viability. Owners would need the Level 2 charger. These are potential plug-in vehicle owners that smart grid advocates would be most concerned about.

Though the PHEV28 could have its battery pack used very effectively, it would drive many miles in charge sustaining mode - as a hybrid. Even when the pack could be fully depleted twice a day, about half of daily miles on average would be in HEV mode. However, the HEV mode of a PHEV28 does have a fuel consumption advantage relative to the charge sustaining mode for an EREV56. And the EREV56, on average, cannot fully deplete the pack twice a day. Using the logic used for the PHEV28 in the 48-80 km group, the EREV56 could on average achieve about $84 \%$ of its miles all electrically. It could achieve about 1.6 charges per day of use. The BEV117 has slightly more range than the average distance traveled in this group. Considering that many of the vehicles travel further than average, a significant fraction of owners would want to add a charge during the day to complete the day's travel, or to reduce range anxiety. With the BEV and the relatively few $\mathrm{km}$ needed to complete the day's travel, there should be flexibility with regard to the time of the daytime charge. This daily distance interval is not long enough to allow a high average for the number of full charges achieved per day of use. The average would be about 1.0, a value for which BEV financial viability at U.S. gasoline and electricity prices would be unlikely.

\section{0-160 km daily distance, daytime charging at work}

Interestingly, three BEV stories recently highlighted by Plug-in America each involved round trip distances to work and back of $96 \mathrm{~km}$, a bit more than the average for this distance group
[29]. In addition, the one example of an EREV56 was also a case where the round-trip distance to work is about the same as the one full charge capability of the vehicle. Thus, Plug-in America is highlighting to its readers at least one full use per day of the capabilities of the chosen plug-in vehicle.

For a BEV117 for this distance bracket, leaving from the house with a full charge and then charging at work would allow nearly all of the owners in the bracket to comfortably meet the day's needs, and would very seldom require afternoon charging. As mentioned earlier, there may be cases where it would be cheaper to charge at work than at the dwelling unit, even when off-peak rates are available. BEV117 owner Tom Moloughney [30] provides his personal example of workplace charging being $60 \%$ cheaper than charging at the house. In this case, the business is a restaurant that he owns, so he is fully aware of the costs of his commercial electricity. He does not say whether the opportunity allowed him to invest less in charging infrastructure. Nevertheless, there would be cases where this possibility exists.

An EREV56 owner arriving at work in the morning refilling the battery pack at work could replace an average of about $91 \%$ of gasoline fueled miles. Should the refilling start before 9 am and use Level 2 charging, the charging could be complete by $1 \mathrm{pm}$ or earlier. A PHEV28 owner doing the same thing could replace about $50 \%$ of gasoline fueled miles, and would also realize savings relative to ownership of an efficient conventional vehicle when driving in HEV mode. For the PHEV28 charging at work starting before 9 am could be done with Level 1 charging and be completed before $1 \mathrm{pm}$.

For a workplace owner, if it is not possible to charge employees for electricity, the question would be what benefits exist in terms of employee satisfaction, reliability, and perhaps employee retention. Could investments in workplace charging pay off in these terms? In the case of [30], who made a commitment to plug-in electric drive, he was able to "internalize" the benefits to his employees, since he was both the owner and employee taking advantage of the low workplace cost of electricity. For society at large, investigations of the cases for and against workplace charging are desirable. Spreading charging time over a greater number of hours than just overnight can allow lower power charge points and may lower cost of infrastructure needed both at dwelling units and workplaces. Perhaps some workplaces will be able to find ways to utilize charge points overnight for their business fleets and during the day for some of their employees. 
This step-by-step discussion of the potential benefits of work place charging does imply that exploration of the ways to accomplish workplace and dwelling unit charging as a system deserve careful attention.

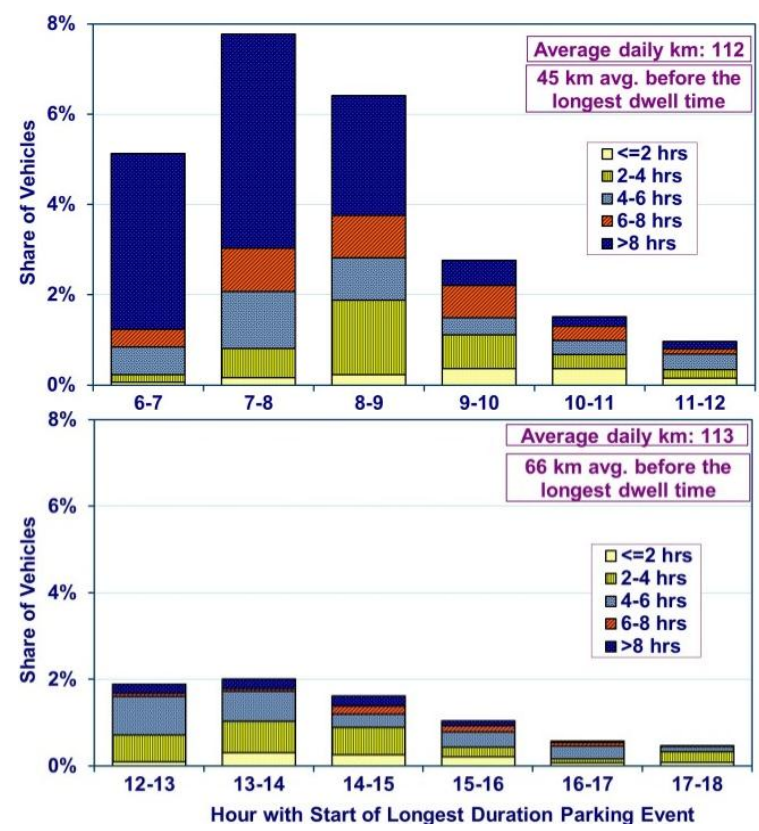

Fig. 7 Share of longest dwell times by hour for work trips within the $80-160 \mathrm{~km}$ group

\section{Conclusions}

The target market for electrification of kilometers by plug-in electric vehicles requires focus on vehicles driven at both advantageous distances per day and appropriate speeds. Prior analyses have emphasized the need for intensive vehicle utilization for plug-in electric powertrains to have lower total costs of operation than conventional and/or hybrid powertrains. However, it has also been observed that the most intensively used vehicles tend to spend a large fraction of distance of travel on limited access highways, where the advantages of conventional drive are greatest, so the target market is probably within a window of daily distances, where too little daily distance makes gasoline powertrains the best choice, while very long daily distances probably make diesel powertrains a better choice. The narrowing of the target markets explored here represents a minority of vehicle owners, but a majority of miles driven. The target market represents intensively used vehicles driven in intra-urban driving and not in rural or intercity driving. Cars and small SUVs used for work commuting appear to be a very important target market for plug-in electric vehicles driven in the selected daily driving ranges (48-80 and $80-160$ $\mathrm{km} / \mathrm{day}$ ). Although there is great enthusiasm for vehicles capable of pure electric operation (BEVs and EREVs), prior financial viability investigations for the U.S. market imply that PHEV options tied to HEV powertrains represent the most cost effective market. This investigation considered whether multiple charges per day might help tip the balance toward EREVs and BEVs instead of PHEVs. In doing so, it was observed that for the two target market daily distance brackets selected the PHEV still appears to be difficult to beat, because its battery pack can be used even more intensively in these circumstances than the EREV or BEV, with lower $\mathrm{kW}$ rating and cost of infrastructure. These results suggest the possibility of mutual reinforcement of related HEV and derivative PHEV designs, a development that may also reduce common component costs by spreading them over more vehicles.

Dynamic pricing advocates for residential customers should realize that the PHEV28 is not another air conditioner, nor is an EREV56 or BEV117. These are trough-filling, load-factor-enhancing appliances whose grid charging should be encouraged. Few of these plug-in vehicles would be at the house on afternoons when summer peak demand occurs. For those that were, owners could much more readily forego the services of those vehicles, than for air conditioning. The PHEV28 is much more likely to use Level 1 charging at about $1.4 \mathrm{~kW}$ while an EREV56 or a BEV117 would be more likely to use $3.3 \mathrm{~kW}$. Thus, on the rare occasions when it did so, the technical ability of the PHEV28 to impose a sharp increase in on peak $\mathrm{kW}$ load would be less than for EREV56 or BEV117 vehicles. Further, the majority of the daytime charging opportunities that PHEV28s would take advantage of would most likely be in the morning at work and could be completed before summer afternoon hours. For any new workplace charge point installations with Level 2 capability, this would also be true for EREV56 vehicles. Finally, existing commercial rate structures which include demand charges would make it likely that summertime on-peak workplace charging would be managed by commercial customers in a way that would deter or even prevent charging on peak.

\section{Acknowledgments}

The authors would like to gratefully acknowledge the sponsorship of David Howell, Team Leader, Hybrid and Electric Systems, Office of Vehicle Technology, U.S. Department of Energy. They are also most appreciative of the ability to share and 
discuss ongoing research with country experts of the Fraunhofer Institute for Systems and Innovation Research ISI of Karlsruhe Germany (David Dallinger and Patrick Plotz), and the Institute of Vehicle Concepts, German Aerospace Center (DLR), Stuttgart Germany (Bernd Propfe). The opportunity for the real time working exchanges arose from German and U.S. participation in the International Energy Agency Hybrid and Electric Vehicle Implementing Agreement Task 15 study of Plug-in Hybrids.

\section{References:}

[1] Dallinger, D., M. Wietschel, Grid integration of intermittent renewable energy sources using price-responsive plug-in electric vehicles, Fraunhofer Institute for Systems and Innovation Research Karlsruhe, Working Paper Sustainability and Innovation No. S 7, 2011.

[2] Santini, D.J., A.D. Vyas, D. Saucedo, and B. Jungers, Where Are the Market Niches for Electric Drive Passenger Cars? Proceedings of the $90^{\text {th }}$ Annual Meeting of the Transportation Research Board, Washington, DC 2011.

[3] General Motors. Volt Owners May Soon Get Charged with Renewable Energy. http://media.gm.com/content/media/us/en/gm/ne ws.detail.html/content/Pages/news/us/en/2012/Ja n/0123_onstar, accessed on 2012-01-23.

[4] Wilson, J. Ford Fusion Energi Plug-In Hybrid Review

http://productsummary.blogspot.com/search/label /Smart\%20TV. accessed on 2012-01-09.

[5] Duvall, M., et al., Comparing the Benefits and Impacts of Hybrid Electric Vehicle Options for Compact Sedan and Sport Utility Vehicles, Report 1006892, Electric Power Research Institute, Palo Alto, California, 2002.

[6] Santini, D.J. Highway Vehicle Electric Drive in the United States: 2009 Status and Issues. Argonne National Laboratory Report ANL/ESD/10-9, 2010.

[7] Gnann, T., P. Plotz and F. Kley. Vehicle charging infrastructure demand for the introduction of plug-in electric vehicles in Germany and the U.S. EVS26, Los Angeles CA, 2012.

[8] Plotz, P. F. Kley and T. Gnann. Optimal Battery Sizes for Plug-in-Hybrid Electric Vehicles. EVS26, Los Angeles CA, 2012.

[9] Moawad, A. et al., Impact of Real World Drive Cycles on PHEV Fuel Efficiency and Cost for Different Powertrain and Battery Characteristics. conference paper EVS24, 2009.

[10] Propfe, B. et al. Cost analysis of Plug-in Hybrid Electric Vehicles including Maintenance \& Repair Costs and Resale Values. EVS26, Los Angeles CA, 2012.

[11] Tal, G. et al. Evaluating Demand for Plug-in Vehicles: A New Car Buyer Study in the San Diego Area. Preliminary Results. UC Davis Plug-in Hybrid Vehicle Research Center. Dec. 20, 2012. http://phev.ucdavis.edu/research/pgesymposium/Gil\%20PGE.pdf accessed on 2012-2-14. [12] Axsen, Jonn, Kenneth S. Kurani, Andrew F. Burke. Are Batteries Ready for Plug-in Hybrid Buyers?. Transport Policy 17 (3), 173 - 182, 2010.

[13] Shiau, C.N. et al. Impact of battery weight and charging patterns on the economic and environmental benefits of plug-in hybrid vehicles. Energy Policy 37 pp. 2653-2663, 2009.

[14] Kurani, K. Taking Stories Seriously. UC Davis Plug-in Hybrid Vehicle Research Center. 2011. http://phev.ucdavis.edu/research/pge-

symposium/Kurani\%20PGE.pdf, accessed on 2012$2-16$.

[15] Zhou, Y, Vyas, A, and Santini, D, 2011, Tracking National Household Vehicle Usage by Vehicle Type, Age and Area In Support of Market Assessments for Plug-in Electric Vehicles, proceedings of the $91^{\text {st }}$ Annual Meeting of the Transportation Research Board, Washington, DC, 2012.

[16] Energy Information Administration, Monthly Energy Review, Energy Information Administration, Washington DC, 2012. http://www.eia.gov/totalenergy/data/monthly/pdf/me r.pdf.

[17] Santini, D.J. et al . Where is the Early Market for PHEVs? World Electric Vehicle Journal Vol. 2, No. 4, pp. 49-98. 2008.

[18] Maitra. A. Plug-In Vehicle Drive Impacts to the Grid. Presented at the Transportation Research Board Environment and Energy Research Conference, Raleigh NC, 2010.

[19] Zimmerman, K. Vehicle Charging Choices for the Volt. Presented at: Getting Plugged In: Electric Drive Community Readiness Training" for Clean Cities Coordinators, Indianapolis, Indiana, 2011.

[20] Zimmerman, K. Volt: Technology-

Infrastructure Presented at: National Alliance for Advanced Battery Technologies Meeting, Dec. 10, 2010. Louisville, KY.

[21] Crosby, M. CPUC Alternative-Fueled Vehicle Rulemaking Policy Context and Proceeding Update. 
Presented at: "Getting Plugged In: Electric Drive Community Readiness Training" for Clean Cities Coordinators, Indianapolis, Indiana, 2011.

[22] S. Thesen, Electrical Service Options for Residential Customer EV \& PHEV Users. CARB ZEV Symposium, Sacramento CA, Sept. 26, 2006.

[23] The Nissan Leaf U.S. owner's manual, http://www.nissan-

techinfo.com/refgh0v/og/Leaf/2012-NissanLEAF.pdf.

[24] The Chevrolet Volt owner's manual, (http://www.chevrolet.com/assets/pdf/owners/ma nuals/2011/2011_chevrolet_volt_owners.pdf.

[25] Elgowainy, A, et al., Impact of plug-in hybrid electric vehicle charging choices in 2030, proceedings of the $91^{\text {st }}$ Annual Meeting of the Transportation Research Board, Washington, DC, 2012.

[26] Vyas, A., D. Santini, and L. Johnson. PlugIn Hybrid Electric Vehicles' Potential for Petroleum Use Reduction: Issues Involved in Developing Reliable Estimates. Transportation Research Record: Journal of the Transportation Research Board No. 2139. Transportation Research Board of the National Academies, Washington, DC, pp. 55-63, 2010.

[27] Davies, J. and K. Kurani. Estimated Marginal Impact of Workplace Charging on Electricity Demand and Charge Depleting Driving, Scenarios Based on Plausible Early Market Consumer's Use of a $5 \mathrm{kWh}$ Conversion PHEV. Aug. $1, \quad 2010$. http://phev.ucdavis.edu/research/TRB\%20Workp lace\%20Charging\%209210.pdf, accessed on 2012-2-16.

[28] Passier, G. et al. Status Overview of Hybrid and Electric Vehicle Technology: Final Report Phase III, Annex VII, IA-HEV, International Energy Agency, Paris, France, 2007.

[29] Plug-in America. Charged Up \& Ready to Roll, $3^{\text {rd }}$ ed. Plug In America, 2012

[30] Moloughney, T. Your New Electric Bill. Pp. 30-31. Plug-in America. Charged Up \& Ready to Roll, $3^{\text {rd }}$ ed. Plug In America, 2012

\section{Authors}

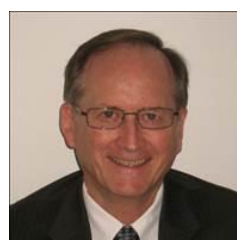

Dr. Danilo J. Santini is a Senior Economist at Argonne National Laboratory. He was chair of the Alternative Fuels Committee of the U.S. Transportation
Research Board from 1996-2002; is now Emeritus. Since 2001 he was a representative for the U.S. DOE to the IEA Implementing Agreement on Electric and Hybrid Vehicles, where he leads a study of PHEVs. In 2010 he was awarded the SAE Barry McNutt prize for Excellence in Automotive Policy Analysis for work on PHEVs.

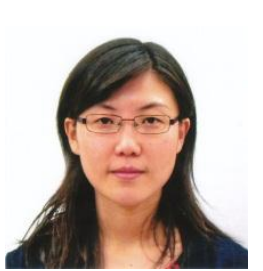

Dr. Yan Zhou received her B.S. degree in Automotive Engineering from Wuhan University of Technology in China in 2003, and a Ph.D. degree in Civil Engineering with a concentration on transportation engineering from Clemson University, South Carolina, in 2010. Currently, she works with the Center for Transportation Research at Argonne National Laboratory. Her research interests mainly include transportation energy demand, market potential and environmental impacts assessment of advanced transportation technologies.

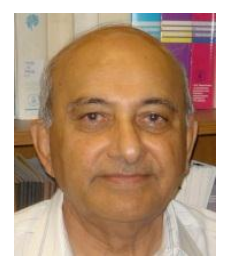

Mr. Anant Vyas is a Transportation Systems Engineer at Argonne's Center for Transportation Research. $\mathrm{He}$ conducts research on transportation demand, market potentials of transportation technologies and materials, and environmental impacts of new technologies. During his 31 years at the Argonne National Laboratory, he has developed and exercised mathematical, simulation, and cost models for evaluation of technologies, materials, alternative fuels, policies, and regulations. Mr. Vyas has written or contributed to over 100 reports, journal articles, conference papers, and presentations. 